\title{
A Model to Explain the TSDC Spectrum of XLPE Insulation in Mid Voltage Cables Based on a Virtual Electrode
}

\author{
J. Belana*, J.A. Diego, J. Orrit and M. Mudarra \\ Departament de Física i Enginyeria Nuclear, \\ Universitat Politècnica de Catalunya, Campus de Terrassa, \\ C/ Colom 11, E08222 Terrassa, Spain \\ *E-mail: juan.belana@upc.edu
}

\begin{abstract}
The analysis by TSDC of a large variety of cables subjected to long annealing times, has resulted in spectra that sometimes show an oscillation of the current peak observed at $99^{\circ} \mathrm{C}$. This process has been observed in some cables only and it is scarcely reproducible. We present an explanation of this behavior based on the formation of charge packets and some other assumptions: charge injection occurs mainly from the negative electro$\mathrm{de}$, and chemical components that act as trapping centers are diffused continously from the semiconducting layers into the bulk during annealing. When the field is applied the negative electrode injects charge that get trapped in these centers, so that a virtual electrode is formed. The electric field created by this layer results in a decrease of the field in the gap between the injecting and virtual electrodes, preventing new charge injection. On the other hand, during polarization the virtual electrode shifts through the insulation bulk towards the opposite electrode. This layer that acts as a virtual electrode becomes neutral by means of recombination when it reaches the opposite electrode, so that the electric field in the region between the electrodes recovers its initial value and the whole process happens again cyclically. This model can explain the observed TSDC oscillating spectra of annealed samples. It can also explain why the oscillations can not be observed in most cases.
\end{abstract}

\section{INTRODUCTION}

Polyethylene (PE) is extensively used in industrial applications such as electrical insulation in medium and high voltage cables. Its glass transition temperature $\left(T_{g}\right)$ lies below room temperature. In cable manufacturing, PE is crosslinked adding chemical initiators such as peroxides. These crosslinkers allow chains to get bound together. DSC studies indicate that the melting of crosslynked polyethylene (XLPE) crystals takes place between 50 and $110^{\circ} \mathrm{C}$. The study of the conductive processes in polyethylene is an issue still open. Dielectric response is due mainly to free space charge although in the manufacturing process some byproducts that have polar character can be added.

The behavior of XLPE along the melting range of temperatures can be tracked by the way of electret forma- tion. Later, their thermal depolarization will give rise to a spectra where the discharge current is recorded in terms of temperature. This technique is known as thermally stimulated depolarization currents (TSDC) [1].

The TSDC spectra obtained after each electret depolarization reflect how the electric field affects the polar and the free space charge mechanisms of the material. TSDC has been extensively applied to the study of dielectric relaxation in a large number of materials, many of them polymers. Presently, there are excellent treateses on this subject [1-4]. The application of this technique to XL$\mathrm{PE}$ gives spectra of certain complexity. These spectra are usually not fully repetitive due to the high sensitivity of the material to thermal history and possible differences between sections of the cable.

Many works on XLPE use segments of cable as samples to perform their study. Using the TSDC technique, a wide peak can be observed in the spectra $[5,6]$. It is placed between 50 and $110^{\circ} \mathrm{C}$ and it has its maximum at about $105^{\circ} \mathrm{C}$. This peak probably results from the release of space charge that was trapped in the crystalline fraction although it could also be associated with crystalline interphases $[7,8]$. It is an structured peak since several overlapped peaks can be observed on it.

There is a minimum between $90^{\circ} \mathrm{C}$ and the maximum at $110^{\circ} \mathrm{C}$. When the sample is annealed at temperatures above $80^{\circ} \mathrm{C}$, this minimum becomes more noticeable until it becomes an homopolar peak placed around $100^{\circ} \mathrm{C}$. This phenomenon occurs faster as the anneling temperature is higher. If the annealing is continued the peak becomes less pronounced, dissapears and finally becomes again heteropolar.

Previous works showed that this homopolar inversion is due to charge detrapping. The charge that arises was previously trapped in defects that diffuse from the semiconductor layers. Infrared spectroscopy (IR) analysis showed that this diffusion of defects into the XLPE bulk takes place in a continuous way when the sample is annealed at temperatures above $80^{\circ} \mathrm{C}$ [9]. Diffused defects act as traps for the charge that is injected from the electrodes during polarization. The application of the electroacustic pulse technique (PEA) has shown asymmetric char- 
ge injection from electrodes and the formation of periodic packets of charge in the case of high electric fields [10]. Although the mechanisms explaining the homopolar behavior were well established in those works, the need of further study to explain the regain of the heteropolar character of the spectra was also evidenced.

Besides this evolution from hetoropolar to homopolar and finally heteropolar behavior of the TSDC spectra, some samples, after long annealings, showed certain trend to regain homopolar behavior. Although this behavior was not observed in all the samples it seem to be related to long annealing times and to specific or particular polarization conditions.

The aim of the present work is to investigate the presence or not of this oscilating behavior in the TSDC spectrum of XLPE cable. For this purpose, several samples were cyclically annealed up to long times and the TSDC spectra measured. A model to explain the oscilating behavior obtained in some cases is discussed.

\section{EXPERIMENTAL}

Cable samples were supplied by General Cable and consisted of a cylindrical insulating XLPE layer with 7 and $13 \mathrm{~mm}$ of inner and outer radii, respectively. The samples had two $1 \mathrm{~mm}$ thick semiconductor layers in contact with the inner and external surfaces of XLPE. The insulating material was crosslinked mixing low density PE with a crosslinking agent (di-t-butyl peroxide) and heating it up to a temperature higher than $200{ }^{\circ} \mathrm{C}$ to produce a vulcanization reaction.

Samples for TSDC measurements consisted in $7 \mathrm{~cm}$ long sections cut from the cable. The semiconductor layers were used as electrodes. To avoid shortcircuits, the external semiconductor layer was partially removed from the sample ends. This left a $2 \mathrm{~cm}$ wide semiconducting strip centered in the sample. The cable core of twisted aluminium wires was used to make contact with the inner semiconductor layer. The contact with the outer semiconductor layer was made by an adjustable metallic clamp.

TSDC measurements were performed in samples annealed up to 47 hours at $120^{\circ} \mathrm{C}$. Experiments were carried out using the non isothermal windowing polarization (NIW) technique. In this method the sample is continuously cooled from an initial temperature $\left(T_{i}\right)$ to the storage temperature $\left(T_{s}\right)$ and the polarizing field is applied during the cooling ramp when the temperature of the sample reaches the polarization temperature $\left(T_{p}\right)$ and switched off a $\Delta T$ below $T_{p}$. The sample remains at $T_{s}$ for a short storage time $\left(t_{s}\right)$ and then it is heated at a constant rate while the TSDC discharge is recorded. In all NIW experiments $T_{i}=120^{\circ} \mathrm{C}, T_{p}=60^{\circ} \mathrm{C}, V_{p}=9 \mathrm{kV}$ (positive inner electrode), $\Delta T=10^{\circ} \mathrm{C}, T_{s}=40^{\circ} \mathrm{C}, t_{s}=5 \mathrm{~min}$ and

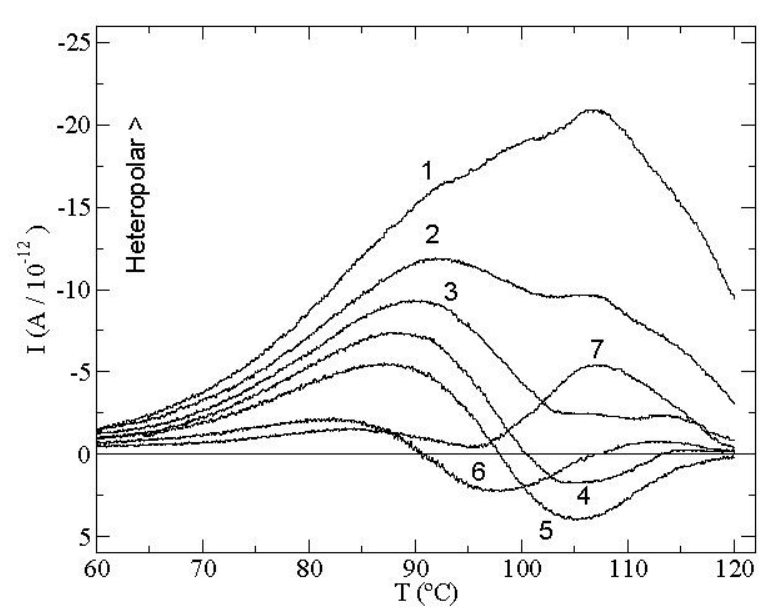

Figure 1: TSDC spectra of XLPE cable showing one oscillation upon annealing at $120^{\circ} \mathrm{C}(1: 0 \mathrm{~h}, 2: 1 \mathrm{~h}, 3: 4 \mathrm{~h}$, 4: $8 \mathrm{~h}, 5: 16 \mathrm{~h}, 6: 32 \mathrm{~h}, 7: 47 \mathrm{~h})$

the cooling and heating rate is $2,5^{\circ} \mathrm{C} / \mathrm{min}$.

\section{RESULTS AND DISCUSSION}

A set of twelve XLPE cable samples were annealed at $120^{\circ} \mathrm{C}$ and measured by TSDC (in the WP or NIW method) from $50^{\circ} \mathrm{C}$ to $120^{\circ} \mathrm{C}$. This process was repeated cyclically for each sample up to an accumulated annealing time of 47 hours. Experimental results showed certain dispersion between samples. In all cases the evolution of the spectrum from heteropolar to homopolar and again heteropolar behavior (hetero $>$ homo $>$ hetero) was observed, although only in three cases the TSDC response regain homopolar behavior after long annealing. Figure 1 is representative of the evolution obtained in nine of the tested samples. We can observe the above mentioned 'hetero > homo>hetero' evolution. These results show as well no trend to regain homopolar character even after 47 hours annealing at $120^{\circ} \mathrm{C}$.

Figure 2 shows the behavior obtained in three of the tested samples. In these cases, after heteropolar sign is regained for the first time (curve 5 in figure 2), current intensity turns homopolar again (curve 6) and, even for longer annealing times heteropolar sign is regained once more (curve 7).

In order to explain this attenuated oscillating behavior we have developed a qualitative model based in our previous studies on XLPE cables. As we reported in previous works, there is a continuous injection of chemical components from the semiconducting external layers (SC) of the cable into the XLPE bulk during annealing at temperature above $80^{\circ} \mathrm{C}$. These chemical components can act as trapping centers, where injected charge can lie. Recent studies in conductivity of cable samples suggest that conductivity in XLPE below $70^{\circ} \mathrm{C}$ occurs preferably by hop- 


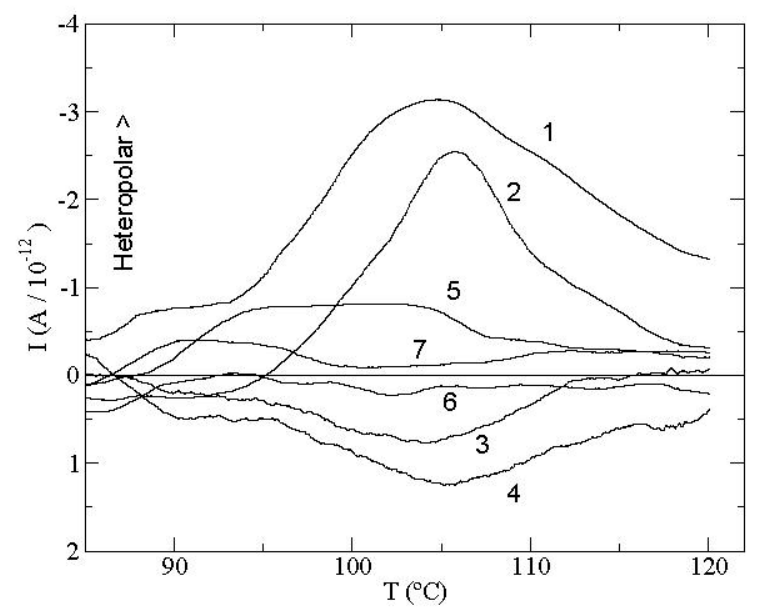

Figure 2: TSDC spectra of XLPE cable showing two oscillations upon annealing at $120^{\circ} \mathrm{C}(1: 0 \mathrm{~h}, 2: 1 \mathrm{~h}, 3: 4 \mathrm{~h}$, $4: 8 \mathrm{~h}, 5: 16 \mathrm{~h}, 6: 32 \mathrm{~h}, 7: 47 \mathrm{~h})$

ping of electrons through trapping centers in the material [11]. Obtained results show, as expected, that an increase in concentration of trapping centers result in an increased conductivity of XLPE.

Our model to explain the observed 'oscillation' of TSDC curves is based in the formation of a 'planar' charged zone within the material. As we will explain, this charged zone is in fact movable, and moves forward through the XLPE bulk during the polarization stage.

When the annealed cable, i/e with a certain density of traps, is polarized, the negative electrode injects charge that initially is trapped close to the XLPE surface (figure 3.a). The presence of this injected charge close to the electrode, results in a significant decrease of the electric field in the electrode-XLPE interphase that eventually prevent further injection of charge. However, the field drifts these electrons to the inner positive electrode and, due to the existence of trapping centers in the material, can move by hopping into the bulk (figure 3.b). While this charged zone moves forward towards the positive electrode, no new charge is injected from the negative electrode as the electric field in zone A of figure 3.b must be small. According to these assumptions, a unique charged planar zone will be formed in the XLPE that moves during polarization up to a certain depth in the bulk. This depth must be related to the mobility of electrons in the material and thus to the trap density (or annealing degree).

To explain how this physical situation can explain the complex TSDC results, we must consider how TSDC technique works. In a typical measurement, both electrodes are grounded once the polarization stage is finished and the compensation charge is formed in each electrode. The sample is then heated and the depolarization current measured. In our case, depending on which electrode is

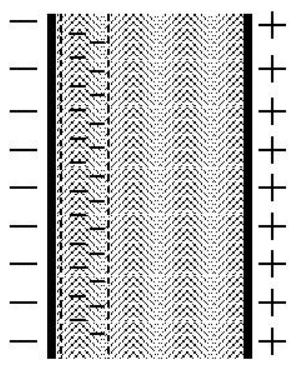

(a)

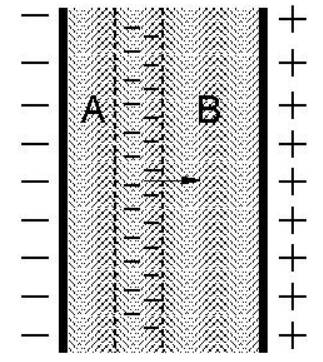

(b)
Figure 3: Schematic representation of the formation (a) and drift (b) of the virtual electrode.

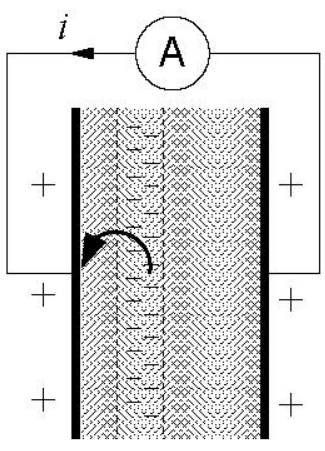

(a)

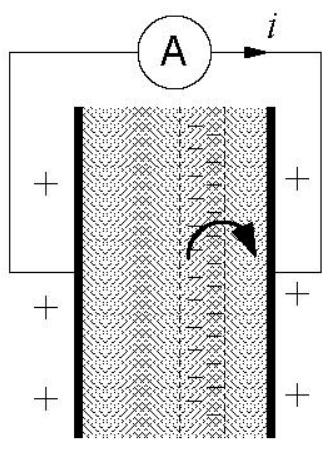

(b)
Figure 4: Schematic representation of the origin of heteropolar sign (a) or homopolar sign (b) in depolarization current.

closer to the charged zone, depolarization current occurs in opposite direction to polarization (heteropolar depolarization current, figure 4.a) or in the same direction to polarization (homopolar depolarization current, figure 4.b). This qualitative model can explain the evolution of TSDC current from heteropolar to homopolar, observed in all cases. As annealing of the sample increases, a higher trap density and thus higher electron mobility is promoted so that the charged zone reaches deeper regions.

We must consider now what happens if the charged zone reaches the inner electrode during the polarization stage. In this case, as the injected charges arrive to the positive electrode, they will be recombined and the electric field in zone A will gradually increase (figure 5.a). This increase will promote the injection of new electrons from the negative electrode resulting in the formation of a new planar charged zone (figure 5.b and 5.c). This process results in the change from homopolar to heteropolar response in the TSDC curve, explaining the experimental behavior observed.

At this point all the process described can, in principle, start again and a continuous oscillation should be obtai- 


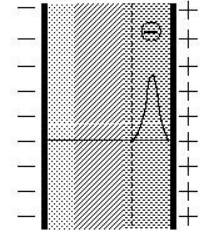

(a)

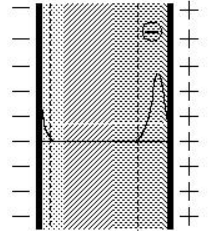

(b)

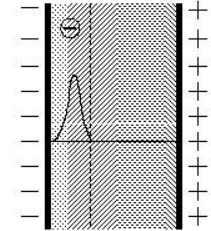

(c)
Figure 5: Schematic representation of recombination of the virtual electrode charges and the formation of a new charged region.

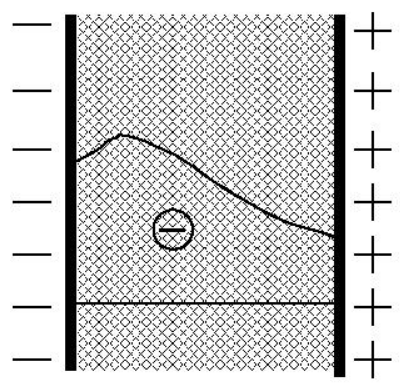

Figure 6: Schematic representation of the formation of a charged zone spread over the sample thickness.

ned. Nevertheless, experiments carried out only show the second oscillation in three of twelve samples, and a great decay in the maximum intensity is observed always after the first oscilation. This fact can be explained as well in the framework of the model assuming that when the trap density is very high, electron drift velocity is high too. This circumstance generates greater dispersion of the charged zone that results in injected charge spread over all the sample thickness (see figure 6). In this case, the depolarization current expected should be heteropolar but certainly not as intense as in the case represented in figure 4.a.

\section{CONCLUSIONS}

TSDC measurements on XLPE from cable samples annealed for long times show, in some cases, the oscillation of the peak observed at $99^{\circ} \mathrm{C}$. The qualitative model presented, based in the formation of a virtual electrode from charge injected during polarization, can explain this complex behavior. This virtual electrode moves during polarization through the material, in a similar way as the reported formation of packets of charge between the electrodes in the case of high electric fields. The increase of mobility of charge carriers, due to the increase in trapping centers density during cable annealing, can explain as well the decay in this oscillation that makes it scarcely observable.

\section{ACKNOWLEDGEMENTS}

This research work has been partially supported by Spanish government project: PTR95.0742.OP and General Cable Co. who kindly supplied cable samples.

\section{REFERENCES}

[1] R. Chen and Y. Kirsh, in Analysis of Thermally Stimulated Processes, chapter 3, pp 60-81, Pergamon Oxford, 1st edition, 1981.

[2] G. M. Sessler, in Electrets, volume 2, chapter 10, pp 41-80. Laplacian, Morgan Hill, CA, 3rd edition, 1999.

[3] B. Hilczer and J. Malecki, in Electrets, chapter 6, pp 285-313. Studies in electrical and Electronical Engineering 14. Elsevier-PWN-Polish Scientific, Warszawa, 1986.

[4] J. V. Turnhout, in J. Thermally Stimulated Discharge of Polymer Electrets, chapter 3, pp 83-96. Elsevier, Amsterdam, 1980.

[5] A. Cavallini, D. Fabiani, G. Mazzanti and G. C. Montanari. IEEE Trans. Dielectr. Electr. Insul. 9 (2002) 514523.

[6] H. Kon, Y. Suzuoki and T. Mizutani. IEEE Trans. Dielectr. Electr. Insul., vol. 3, pp 380-385, 1996.

[7] I. Tamayo, J. Belana, J. C. Cañadas, M. Mudarra, J. A. Diego and J. Sellarès, J. Polym. Sci. Part B: Polym. Phys., vol. 41, pp 1412-1421, 2003.

[8] J.A. Diego J. Belana, J. Orrit, J. Sellarès, M. Mudarra and J.C. Cañadas, J. Phys. D: Appl Phys., vol 39, pp 1932-1938, 2006.

[9] I. Tamayo, J. Belana, J. A. Diego, J. C. Cañadas, M. Mudarra and J. Sellarès, J. Polym. Sci. Part B: Polym. Phys., vol. 42, pp 4164-4174, 2004.

[10] N. Hozumi, H. Suzuki,T. Okamoto,K. Watanabe and A. Watanabe, . IEEE Trans. Dielectr.Electr.Insul., vol. 1, $\mathrm{n}^{\circ} 6$, pp 1068-1076, 1994.

[11] N. Zeobuchi, T.G. Hoang and A. Bui, J. Appl. Phys., vol 81(5), pp2363-2369, 1997. 